\title{
Simple versus cooperative relaxations in complex correlated systems
}

\author{
J. F. Mano ${ }^{\text {a) }}$ \\ Department of Polymer Engineering, Universidade do Minho, 4800 Guimarães, Portugal \\ S. Lanceros-Méndez \\ Department of Physics, Universidade do Minho, 4710 Braga Codex, Portugal
}

(Received 27 March 2000; accepted for publication 26 October 2000)

\begin{abstract}
A method for investigating the nature of thermally activated relaxations in terms of their cooperative character is tested in both polymer and low molecular weight crystal systems. This approach is based on analysis of the activation entropy in order to describe thermally activated relaxations. The betaine arsenate/phosphate mixed system of low molecular weight crystals was selected for investigation because pure compounds of this system show ferro-/antiferroelectric phase transitions and the mixed crystals undergo different kinds of relaxation processes involving both dipole-dipole and dipole-lattice interactions. The polymer chosen was a side chain liquid-crystalline polysiloxane, which shows the $\beta$-relaxation characteristic of disordered systems and amorphous materials. The cooperative versus local character of the relaxations is described in terms of "complex" and "simple" relaxations based on calculations of the activation entropies. The initial assumptions of the theory, as well as the resulting equations, were found to be applicable to the systems studied. (C) 2001 American Institute of Physics. [DOI: 10.1063/1.1334937]
\end{abstract}

\section{INTRODUCTION}

The study of molecular mobility in the solid state is one of the most fascinating areas in physics. The scope of investigation covers a variety of areas, such as solid-state physics, biology, chemistry, and polymer/colloid science. The onset of molecular motion modes occurs over specific temperature ranges and time scales and determines the change in material properties. These two parameters are usually correlated. For simple thermally activated processes the relationship may be described by the Arrhenius equation.

$$
f=f_{0} \exp \left(-E_{a} / R T\right),
$$

where $f$ is the frequency (the characteristic time $\tau$ is $1 / 2 \pi f$ ), $E_{a}$ is the activation energy, $f_{0}$ is a pre-exponential factor, and $R$ is the gas constant. A similar relationship is derived from the theory of absolute reaction rates, proposed by Eyring, ${ }^{1}$

$$
f=\frac{k T}{2 \pi h} \exp (\Delta S / R) \exp (-\Delta H / R T),
$$

where $\Delta H$ and $\Delta S$ are the activation enthalpy and entropy, respectively. The thermokinetic parameters of Eqs. (1) and (2) can be obtained by fitting $\ln f$ vs $1 / T$ and $\ln (f / T)$ vs $1 / T$ data.

However, it is not possible to describe some relaxation processes with Eqs. (1) and (2). The mechanisms involved during the glass transition, which occurs in all glass forming systems, are probably the most important examples. On the other hand, the temperature dependence of the kinetics of localized modes, which involve small correlation lengths, is usually thermally activated. For example, $\beta$ relaxation, de-

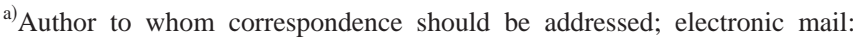
jmano@eng.uminho.pt
}

scribed well by Johari and Goldstein, ${ }^{2}$ is assumed to follow the Arrhenius equation. This process is usually observed in disordered systems in the glassy state and the experimental data are interpreted as local free volume fluctuations. Lower temperature (or higher frequency) relaxations of this kind (for example, the $\delta$ and $\gamma$ relaxations) have been observed in polymer systems. ${ }^{3}$ Moreover, local and vibrational modes observed in a large variety of materials are also thermally activated processes.

Starkweather proposed a method for investigating the nature of thermally activated relaxations in terms of their cooperative character. ${ }^{4,5} \mathrm{He}$ designated as "simple relaxations" those involving the motion of small groups of atoms with little interaction with other neighboring atoms. This class of relaxations should have activation entropies, $\Delta S$, near zero. Other processes having large activation entropies were designated as "complex relaxations" or "cooperative relaxations." Those should involve a range of related motions with extensive intra- and intermolecular interactions. The assignment of the cooperative character is thus carried out by comparing the activation energy of the process with the corresponding activation energy of a "zero-entropy" relaxation. The construction of the zero-entropy prediction begins with the comparison between $E_{a}$ and $\Delta H$.

From a comparison between the Arrhenius and the absolute reaction rates theories one obtains ${ }^{6}$

$$
E_{a}=\Delta H+R T-P \Delta V .
$$

Here $\Delta V$ is the activation volume and $P$ is the pressure. Note that, by considering all the terms of Eq. (3) the comparison between $E_{a}$ and $\Delta H$ could be used for calculating the activation volume if the pressure is known. As a first approximation we will neglect the last term in Eq. (3) $(-P \Delta V)$ because of its low magnitude. Equations (1) and (2) may now be rewritten as 


$$
\begin{aligned}
E_{a} & =R T[1+\ln (k T / 2 \pi h f)]+T \Delta S \\
& =R T[22.92+\ln (T / f)]+T \Delta S .
\end{aligned}
$$

For relaxations having zero activation entropy, Eq. (4) reduces to

$$
E_{a}=R T[22.92+\ln (T / f)] .
$$

Starkweather compared the plot of $E_{a}$ vs $T$ from experimental results involving polymer systems with Eq. (5) for a given frequency. According to him, an evident deviation of the experimental results from the theoretical curve is ascribed to processes with a cooperative character. If agreement is observed among the data, the process is considered simple and noncooperative. Most of the low temperature relaxations $(\beta, \delta, \gamma$, etc. ) discussed by this author showed more or less a deviation from the zero entropy line in a $E_{a}$ vs $T$ plot. Among a large number of studied materials, only a few $\beta$ relaxations were found to be nearly pure noncooperative processes. ${ }^{4,5}$

In this work we aim to extend this procedure to analyze dynamic processes occurring in other materials, namely, those with a low molecular weight. First we will apply this method to a liquid crystalline polymer and then to the mixed crystal system betaine arsenate/phosphate.

It should be stressed that the systems chosen are very different in nature, both from the chemical and the crystallographic/morphologic points of view. However, the approach used to describe and characterize their relaxational processes goes beyond such differences, reinforcing the idea of the universality of relaxational phenomena.

\section{APPLICATION TO A LIQUID CRYSTALLINE POLYMER}

Liquid crystalline polymers have been widely studied over the last 20 years because of their interesting electrooptical properties as well as their many physical properties of conventional polymers. ${ }^{7,8}$

The molecular motion in side chain liquid-crystalline polymers (LCPs) have been extensively investigated by dielectric relaxation spectroscopy (DRS) (Ref. 9, and references therein). Typically two main relaxation mechanisms, alpha $\alpha$ and $\delta$, were identified in the liquid-crystalline or isotropic phases and attributed to complex modes of motion involving the mesogenic side groups or the main chain (Ref. 10 , and references therein).

In the glassy state a $\beta$ relaxation mechanism may also be observed in side chain LCPs. Most of those materials have a polar mesogenic group with phenyl groups in the core, linked to the main chain by a flexible chain with, typically, 3-10 carbon atoms. Work using DRS investigating side chain LCPs, with- $\left(\mathrm{CH}_{2}\right)_{n}-R_{1}-\varnothing-\mathrm{COO}-\varnothing-R_{3}$ side groups $\left(R_{1}\right.$ and $R_{3}$ are polar groups and $\varnothing$ is a $p$-substituted biphenyl group), reported one ${ }^{11-15}$ or two ${ }^{16} \beta$ relaxations (termed $\beta_{1}$ and $\beta_{2}$ ). In all cases these relaxations were attributed to rotations in the side group, mainly due to the dipoles in $R_{1}$ and-COO-. In spite of the chemical difference in the lateral groups of these materials, the authors of Refs. 11-15 found activation energies for all the $\beta$ processes between 12 and $13.7 \mathrm{kcal} \mathrm{mol}^{-1}$. The authors of Refs. 11-14 attributed the $\beta$

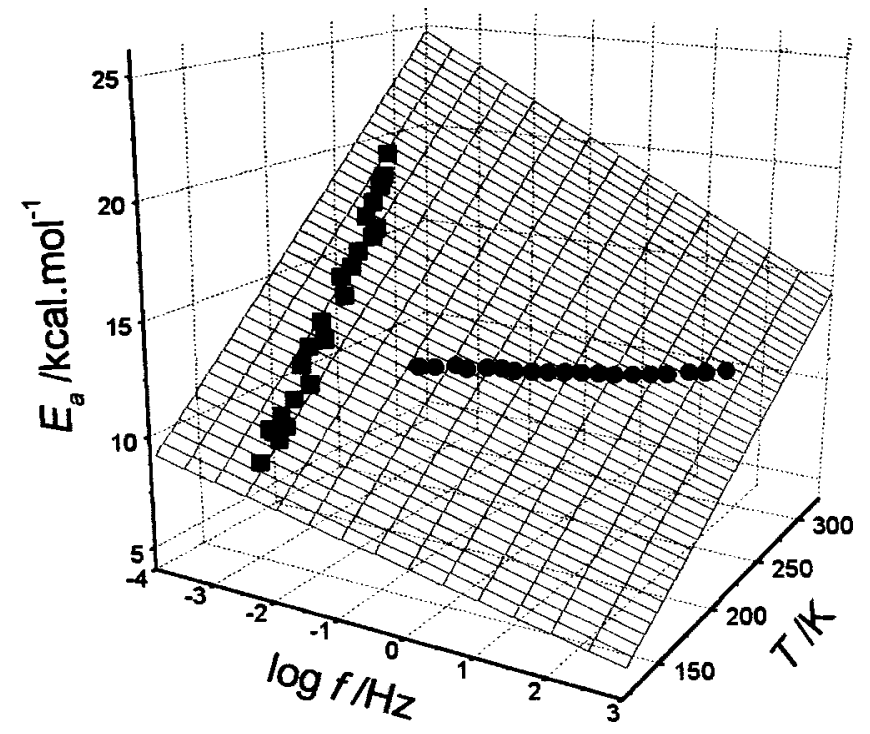

FIG. 1. Relaxation plot with the TSDC (squares) and dielectric relaxation (circles) results of the $\beta$ relaxation of LCP1. The surface plot corresponds to the activation energy for the zero activation entropy as a function of temperature and frequency, according to Eq. (5).

process they observed to rotation of the phenyl benzoate group. On the other hand, Colomer et al. ${ }^{16}$ associated the $\beta_{1}$ process (with an activation energy of $14 \mathrm{kcal} \mathrm{mol}^{-1}$ ) to the same rotation and the $\beta_{2}$ process to the rotation of the $R_{1}$ $=-\mathrm{COO}-$ group, with an activation energy of 9.6 $\mathrm{kcal} \mathrm{mol}^{-1}$. Note that the $\beta_{1}$ and $\beta_{2}$ processes appear to overlap in the frequency range of $10^{2}-10^{5} \mathrm{~Hz}$, which provides a possible explanation as to why the authors of Refs. 11-15 could not differentiate between these two relaxations.

In recent work ${ }^{17}$ a low frequency dielectric related technique, the thermally stimulated depolarization currents (TSDC) technique was used to separate these two contributions from the overall $\beta$ relaxation. An advantage of the TSDC technique is the possibility of using a thermal cleaning experimental procedure to decompose a complex relaxation into discrete contributions. These individual processes often follow Arrhenius behavior for low temperature relaxations, i.e., one can assign an activation energy and a preexponential factor to each component.

From a phenomenological point of view, the $\beta$ relaxations observed in those materials are very similar to those observed in other glass forming polymers. Thus, any conclusions made from studying the $\beta$ relaxation of side chain LCPs may be extrapolated to the same processes in other polymeric materials.

One of LCPs studied reported in Ref. 17 (LCP1), was also studied using DRS. ${ }^{18}$ This material has a polysiloxane backbone and each repeat unit has a side chain with the structure- $\left(\mathrm{CH}_{2}\right)_{6}-\mathrm{O}-\varnothing-\mathrm{COO}-\varnothing-\mathrm{COOCH}_{2} \mathrm{CH}\left(\mathrm{CH}_{3}\right)\left(\mathrm{C}_{2} \mathrm{H}_{5}\right)$. As expected, the $\beta$ relaxation studied by TSDC and DRS gave similar results in terms of both activation energy and temperature location of the process when analyzed using a $\log \tau$ vs $1 / T$ plot. $^{19}$

In Fig. 1 (surface plot) the activation energy is plotted against temperature and frequency according to Eq. (5). Thus, if the experimental data agree with this representation, 


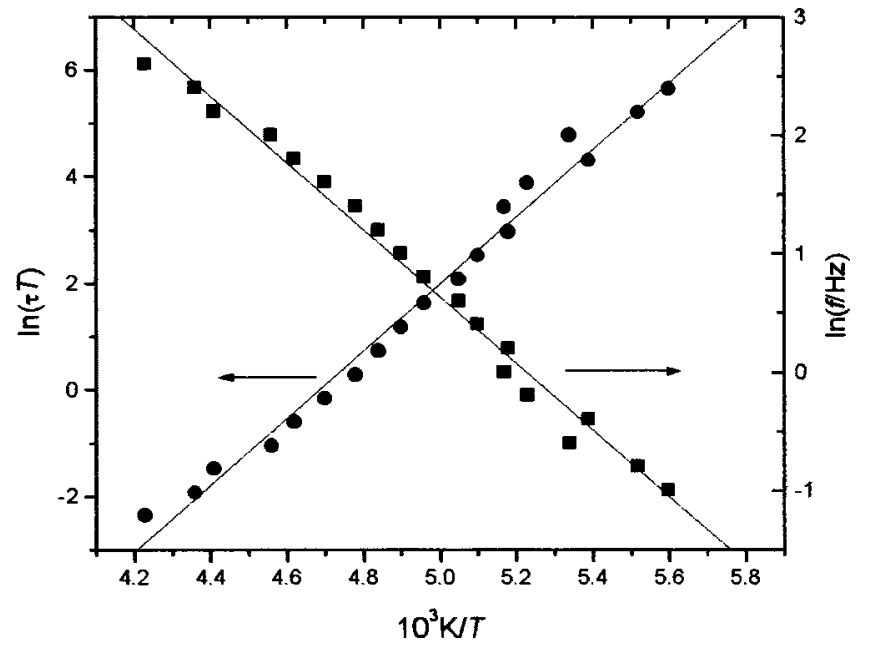

FIG. 2. Arrhenius diagram (squares) and Eyring plot (circles) for the $\beta$ relaxation of LCP1 obtained by dielectric relaxation. The solid lines are the linear fittings of the two representations.

according to Starkweather, the processes involved should be local and noncooperative.

The results of LCP1 obtained by DRS and TSDC (thermal sampling results) are also given in Fig. 1. Ignoring any prospective error, it is possible to conclude that the $\beta$ process should be assigned upon first analysis to a simple noncooperative process. It must be mentioned in this context that for the TSDC results the activation entropy was calculated for each thermal component sampled. It was found that the values fluctuated around $\Delta S=0$ (from $\sim-10$ to $\sim 10$ cal K $\left.{ }^{-1} \mathrm{~mol}^{-1}\right){ }^{17}$

The Arrhenius and Eyring plots of the DRS results on the LCP1 system are shown in Fig. 2. The adjustable parameters obtained from the fits were $E_{a}=12.90 \mathrm{kcal} \mathrm{mol}^{-1} ; f_{0}$ $=5.52 \times 10^{14} \mathrm{~Hz}, \quad \Delta H=12.50 \mathrm{kcal} \mathrm{mol}^{-1}, \quad$ and $\quad \Delta S$ $=11.3 \mathrm{cal} \mathrm{K}^{-1} \mathrm{~mol}^{-1}$. Note that from DRS $\Delta S$ is not zero. However, considering the system under study, the value obtained is not very high (note, for example, that we found activation entropies close to $250 \mathrm{cal} \mathrm{K}^{-1} \mathrm{~mol}^{-1}$ for the same material near $T_{g}$ by TSDC).

The deviation of the DRS results from the zero-entropy prediction can also be observed in a $E_{a}$ vs $T$ plot. In Fig. 3 the closed circle shows the location of the relaxation at $f$ $=1 \mathrm{kHz}(T=240 \mathrm{~K}$ calculated by the Arrhenius equation). The solid line represents the location of any relaxation obeying $\Delta S=0$ at $1 \mathrm{kHz}$, plotted according Eq. (5). The difference between the experimental result of the $\beta$ relaxation of LCP1 and the zero-entropy prediction is $2.64 \mathrm{kcal} \mathrm{mol}^{-1}$ and must be the $T \Delta S$ term in Eq. (4). Differences of this order were also found in nearly noncooperative relaxation observed in some amorphous polymers. ${ }^{4,5}$ It is difficult to assign the relaxation to a completely noncooperative process because it is not zero but, from the TSDC data and by considering the usual experimental errors, one cannot attribute the origin of this process to a strong complex translational/ rotational mechanism. We can predict that there is certain complexity in this process, i.e., in terms of Adam-Gibbs theory, any configurational change only occurs with configu-
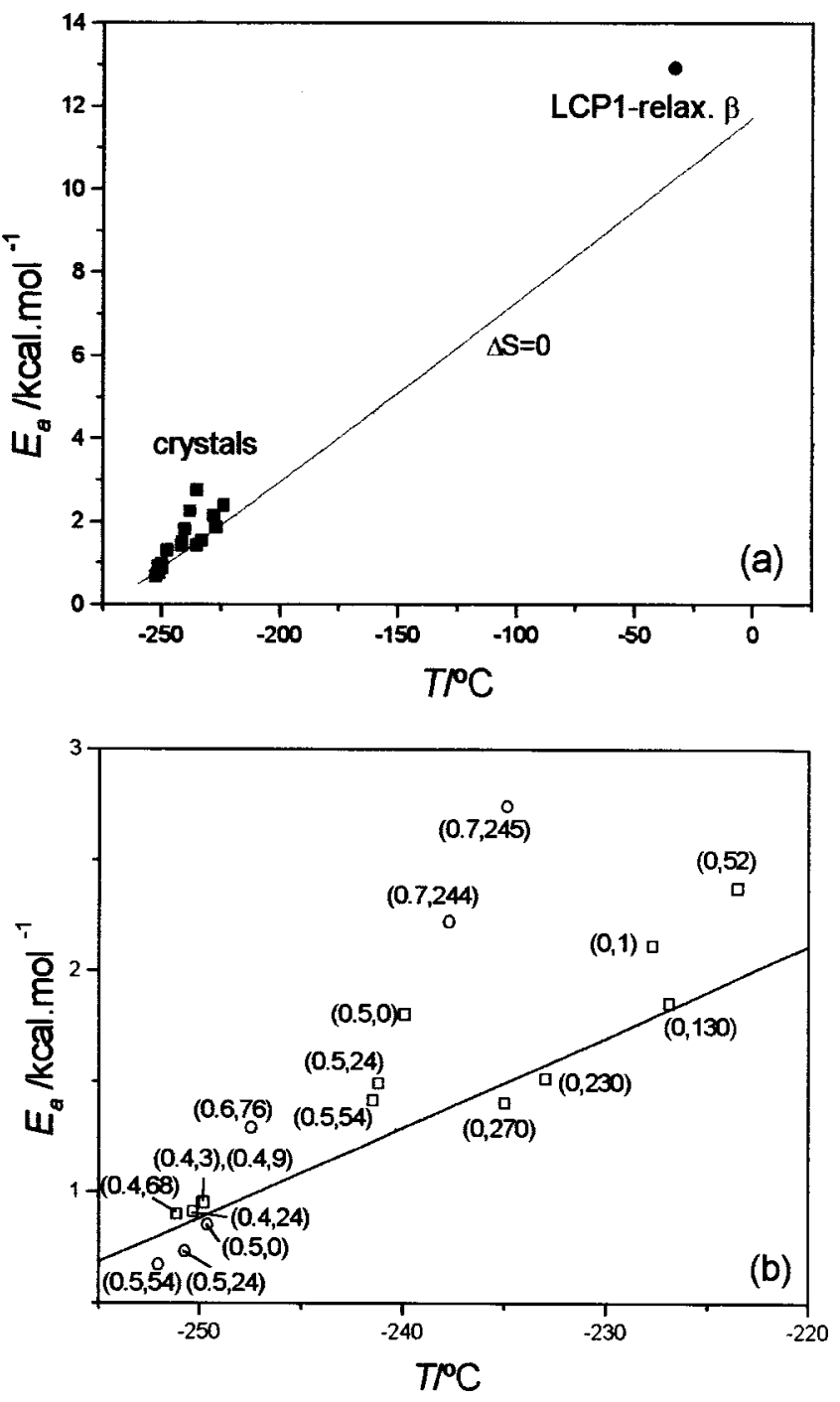

FIG. 3. (a) Activation energies as a function of temperature $(1 \mathrm{kHz})$ for the results on the $\mathrm{BA}_{x} \mathrm{BP}_{1-x}$ crystals (closed squares) and for the $\beta$ relaxation of LCP1 (closed circle). The solid line is the zero-entropy prediction at $f$ $=1 \mathrm{kHz}$. The extent to which the activation energy exceeds the zeroentropy value is proportional to $T \Delta S$ and, consequently, to the degree of cooperativity of the relaxation. (b) Magnification of the graphics in (a), covering the temperature region of the crystal results. The parentheses close to the symbols indicate the concentration of betaine arsenate and the pressure in MPa.

rational rearrangements in the vicinity throughout a volume with a certain correlation length.

\section{APPLICATION TO MIXED CRYSTAL SYSTEMS: BETAINE ARSENATE/PHOSPHATE}

Several compounds of the amino acid betaine $\left[\left(\mathrm{CH}_{3}\right)_{3} \mathrm{NCH}_{2} \mathrm{COO}^{-}\right]$and inorganic acids display interesting structural phase transitions. Ferroelectric (FE), antiferroelectric $(\mathrm{AF})$, ferroelastic, and modulated (commensurate and incommensurate) phase have been found. ${ }^{20}$

Betaine arsenate $\left(\ldots \cdot \mathrm{H}_{3} \mathrm{AsO}_{4}, \mathrm{BA}, \mathrm{FE}\right)$ and betaine phosphate $\left(\ldots \cdot \mathrm{H}_{3} \mathrm{PO}_{4}, \mathrm{BP}, \mathrm{AF}\right)$ are structurally very closely related and mixed crystals are formed accordingly: $\mathrm{BA}_{x} \mathrm{BP}_{1-x}, 0 \leqslant x \leqslant 1$. These crystals have been studied by means of dielectric relaxation spectroscopy under hydrostatic 


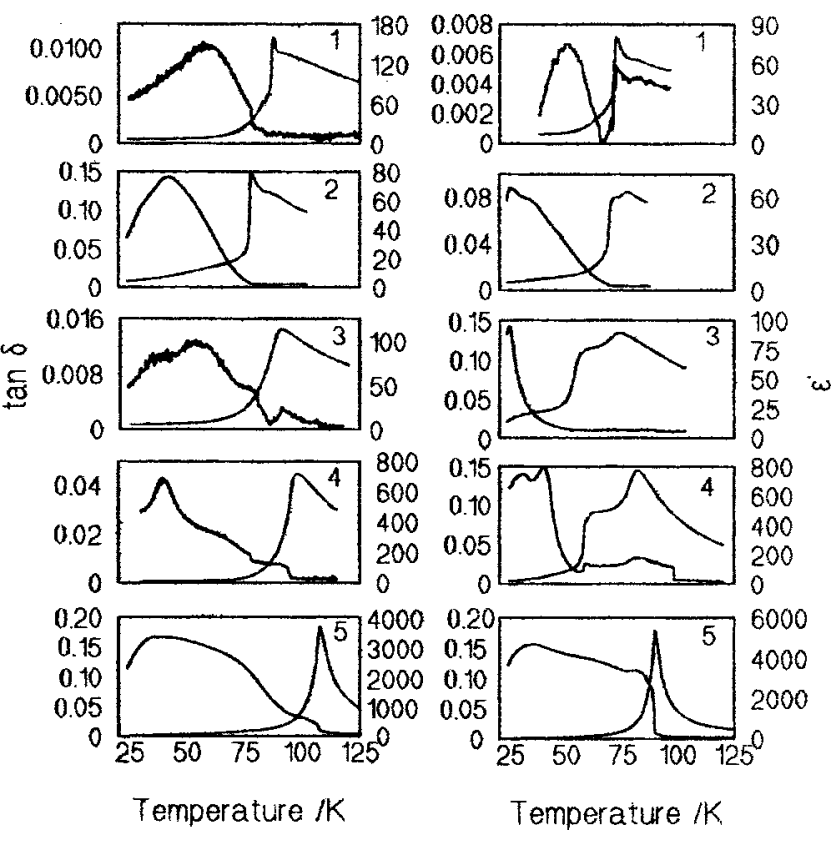

FIG. 4. Typical $\epsilon^{\prime}(T)$ and $\tan \delta(T)$ curves of $\mathrm{BA}_{x} \mathrm{BP}_{1-x}$ for different concentrations; $x=0.07(1), 0.24(2), 0.62(3), 0.73$ (4), and 0.86 (5) at normal (left) and high (right) pressures. $f=100 \mathrm{kHz}$. For each concentration, the curves showing peaks at higher temperatures correspond to the $\epsilon^{\prime}(T)$ data.

pressure (Fig. 4) and external bias field and by measurements of the dielectric hysteresis loops and Raman and IR spectroscopy. ${ }^{21-24}$

The temperature-pressure ${ }^{22,24}$ and temperature-electric bias field ${ }^{21,24}$ phase diagrams of several concentrations have been depicted and the different phases analyzed and characterized. The phase diagrams were divided into two main regions: a transition into a polar-ordered phase (FE, AF, or mixed) at higher temperatures, and a further lower temperature region characterized by different relaxational processes, some probably relating to a glassy phase. This behavior is summarized in the temperature-concentration phase diagram shown in Fig. 5.

The low temperature region in the different concentrations is characterized by strong anomalies in both $\varepsilon^{\prime \prime}(T)$ and $\tan \delta(T)$ which display a strong frequency dispersion also observed in $\varepsilon^{\prime}(T)$ (see Fig. 4). ${ }^{21-24}$ The Arrhenius versus Vogel-Fulcher behavior of the different anomalies in $\tan \delta(T)$ and $\varepsilon^{\prime \prime}(T)$ was investigated. Two mechanisms of relaxation were observed from the phase diagram. Their importance changed, depending on the concentration, which determined the different interaction mechanisms.

(1) A relaxation fitted with an Arrhenius formula in pure $\mathrm{BP}$ with high values in the hindering barrier indicates a dipole-lattice interaction as responsible for this relaxation process. Random fields seem to suppress this process at a low concentration of impurities. ${ }^{23,24}$

(2) Competing interactions appear with increasing $x$. These interactions induce collective processes with different order tendencies. At low temperatures within the AF region of the phase diagram two relaxations appear: the collective relaxation of the dipoles into a dipole glass phase due to competing interactions, and local freezing due to the interac-

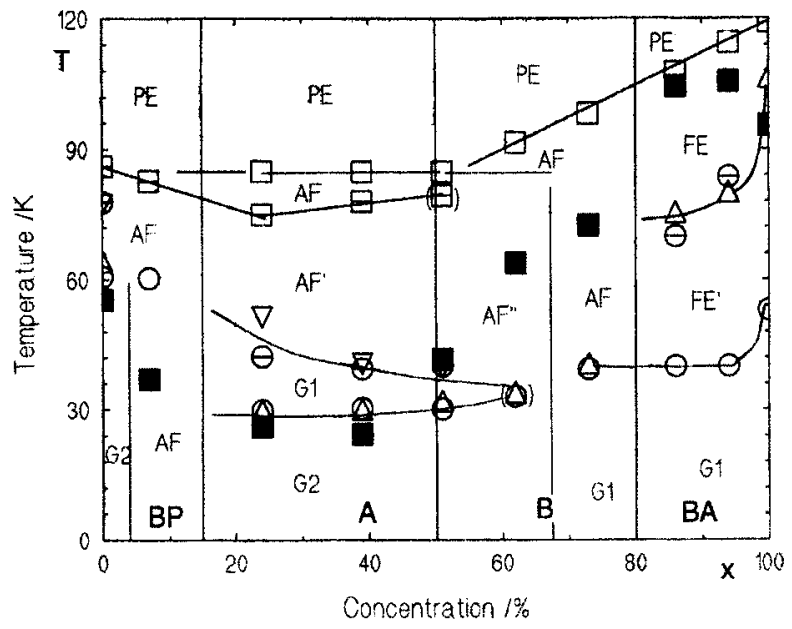

FIG. 5. Transition temperatures vs concentration diagram of $\mathrm{BA}_{x} \mathrm{BP}_{1-x}$ for the whole concentration region at $0.1 \mathrm{MPa}$ and $f=100 \mathrm{kHz}$. The open squares correspond to the anomalies in $\epsilon^{\prime}(T)$ whereas the circles correspond to the anomalies in $\tan \delta(T)$. The triangles represent anomalies in $\epsilon^{\prime \prime}(T)$. The brackets in $\tan \delta(T)$ indicate anomalies much smaller than the others so that we could not observe the corresponding anomaly in $\epsilon^{\prime \prime}(T)$. The brackets in $\epsilon^{\prime}(T)$ indicate a small shoulder more than a well-defined anomaly AF: antiferroelectric, $\mathrm{AF}^{\prime}$ : coexistence of $\mathrm{FE}$ and $\mathrm{AF}$ components, $\mathrm{AF}^{\prime \prime}$ : coexistence of two AF components, G1: VF, i.e., collective relaxation, G2: A, i.e., single-ion relaxation, $\mathrm{FE}$ : ferroelectric, $\mathrm{FE}^{\prime}$ : $\mathrm{FE}$ with the domain freezing process. Solid lines are drawn to guide the eye

tion with the lattice. For $0.15<x<0.50$ coexistence of the two relaxation processes occurs before final freezing into the dipole glass phase.

(3) For $0.50<x<0.8$ the relaxation mechanisms depend strongly on small amounts of impurities and on external parameters, such as hydrostatic pressure or electric bias field. The relaxation is dominated by Arrhenius behavior at lower concentrations and by Vogel-Fulcher behavior at intermediate and higher ones. With increasing pressure both mechanisms appear at low and high concentrations, but only Vogel-Fulcher type relaxation is seen at intermediate ones. Whereas the fitted parameters (activation energy, cut-off frequency, and Vogel-Fulcher temperature) obtained for the other regions in the phase diagram fit together well, ${ }^{23,24}$ the parameters in this region show higher values in both $E_{a} / k_{B}$ and $f_{0}$. Further, at $x \approx 0.60$ a crossover between the local and the collective process seems to occur: for $x<0.60$ the Vogel-Fulcher-type process occurs at higher temperatures than the Arrhenius-type process; the opposite behavior is found for $x>0.60$. Local relaxation, on the other hand, disappears in the FE phase and therefore appears to be related to AF order.

(4) The FE region of the phase diagram is dominated by two relaxations when fitted with a Vogel-Fulcher formula. One of them is related with freezing of the domain structure and the other with freezing of the dipoles inside these domains. The first mechanism depends strongly on external influences like the electric field, hydrostatic pressure, and concentration, whereas the second one is independent of all these parameters. $^{22}$

With respect to the microscopic origin of the phase behavior, the Raman measurements and the analysis with the quasione- 
dimensional Ising model ${ }^{24}$ give strong evidence that both the $\mathrm{PO}_{4} / \mathrm{AsO}_{4}$ tetrahedra and the betaine molecule $(\mathrm{C}-\mathrm{H}$ vibrations) are responsible for the temperature behavior of each sample and that the degrees of freedom of the tetrahedra account for the changes with respect to the concentration.

For a deeper understanding of the nature of the relaxations we analyzed their cooperative character within the theory proposed in Refs. 4 and 5. The first step was to separate the relaxations fitted with an Arrhenius formula from the ones fitted with a Vogel-Fulcher formula, which already indicates the existence of cooperative behavior and a nonthermally activated process. Among relaxations of this type are those found in the FE part of the phase diagram.

For the relaxations showing Arrhenius behavior, the activation energy and the cut-off frequency for the various relaxations were obtained from fitting the Arrhenius formula to the experimental data. ${ }^{21,24}$ Using the same formula, the temperature at which the relaxation occurred for a frequency of 1 $\mathrm{kHz}$ was calculated. Finally, the activation energy versus temperature was plotted (Fig. 3) for $1 \mathrm{kHz}$, together with the line of zero-entropy calculated from Eq. (5). This process was used for the relaxations that appear in the concentrations $x=0.0,0.39,0.51,0.62$, and 0.73 , all on the AF side of the temperature-concentration phase diagram. The sample with $x=0.24$, not shown in Fig. 3, has behavior similar to the sample with $x=0.39$.

In the previous discussions of the $\mathrm{BA}_{x} \mathrm{BP}_{1-x}$ system, two mechanisms were pointed out as responsible for the different relaxations, dipole-dipole interaction and dipolelattice interaction. These mechanisms are clearly distinguished in the $x=0.24,0.39$, and 0.51 samples.

In the $\mathrm{AF}$ part of the phase diagram, the relaxations at higher concentrations, $x=0.62$ and 0.73 , change from Vogel-Fulcher to Arrhenius behavior by applying external pressure. These high pressure Arrhenius relaxations show a large activation entropy, demonstrating a highly cooperative character. The change from one type of relaxation to the other implies that the Vogel-Fulcher temperature goes to zero as pressure increases.

More interesting and helpful in elucidating the nature of the different processes is the analysis of the relaxations appearing at intermediate concentrations within the $\mathrm{AF}$ region of the phase diagram. The relaxations fitted with an Arrhenius formula at normal pressure experience two different types of behavior under pressure that can reflect the microscopic nature of the process. The single relaxation appearing in pure BP shows some entropy at zero and low pressures ( $p$ $<100 \mathrm{MPa}$ ) but this disappears upon increasing the external pressure. This behavior points to an increase of dipolelattice interaction and, consequently, a decrease of dipoledipole interactions, thus reducing the cooperativity of the system. By increasing the amount of impurities this relaxation disappears, since the effect of increasing concentration produces a similar (but not identical) effect as the increase of pressure. It is assumed that impurities also increase dipolelattice interactions due to the appearance of random fields that hinder the dipoles from following the applied field. ${ }^{22}$

The effect of impurities as further analyzed in the $x$ $=0.39$ sample and the low temperature relaxation in the 0.51 sample (open squares in Fig. 3). Both relaxations are, within experimental error, entropy-free relaxations, i.e., local relaxations with no cooperative character. This behavior is somewhat independent of pressure and can be ascribed to strong dipole-lattice interaction. These relaxations can be considered to have the same origin as the one appearing in pure BP.

The higher temperature relaxation in the 0.51 sample (open circles in Fig. 3) already shows behavior which will lead to the development of a new FE phase and will be a characteristic of this region of the phase diagram. Here there are no longer any impurities, but the betaine arsenate fraction of the crystal starts to develop interactions and a cooperative character. This cooperative character is ascribed to a dipoledipole interaction that is severely disturbed by the increase of external pressure. The effect of pressure produces an increase of dipole-lattice interactions and an increase of the local character of the relaxation. In Fig. 5 we observe that having a relatively high activation entropy at low pressure leads to a linear decrease in relaxation upon applying external pressure. This behavior is the same as the one observed in the 0.62 and the 0.73 samples. These samples display relaxations with Vogel-Fulcher or cooperative behavior at low and medium pressures that changes to Arrhenius relaxation at higher pressures. This high pressure relaxation retains nevertheless, the characteristic cooperativity and consequently shows a large configuration entropy.

\section{CONCLUSION}

Two very different systems from the microscopic and chemical points of view have been analyzed with the same theoretical tools in order to stress the universal character of the method proposed by Starkweather. ${ }^{4,5}$ This theoretical framework assumes that the activated entropy associated with a given relaxation process will define its cooperative or noncooperative character. The parameters used in this procedure are simply the activation energy of the relaxation and its location in the temperature axis for a given frequency. With this theory the activation energy that is usually used only to describe the energy barrier between relaxed and unrelaxed states may be also used to quantify the cooperativity of the molecular mobility associated with the relaxation studied. This will allow quantitative analysis of the variation of the cooperativity of a given relaxation under different conditions, e.g., composition and mechanical and electrical environment. In the case of polymer systems the theory has already been used in the study of cooperative and noncooperative relaxation. In this article we have shown that the theory can also be successfully applied to single crystals. Within the scope of this analysis, the relaxations appearing in single and mixed crystals can also be divided into cooperative and noncooperative depending on the configurational entropy associated to these relaxations. This system is especially suitable for verifying the assumption of the theory, since we were able to compare the degree of cooperativity of the materials with different compositions studied at different pressures. The results are not only in agreement with the initial predictions about the nature of the relaxations (dipole-dipole or dipole-lattice interactions), but have also 
provided a deeper understanding of the modifications of the mechanisms underlying the molecular motion involved in those processes under different external conditions. The analysis may be extended to other systems, e.g., relaxor ferroelectrics, proton glasses, etc., in order to further explore the universality of the present approach.

${ }^{1}$ E. Eyring, J. Chem. Phys. 4, 283 (1936).

${ }^{2}$ G. Johari and M. Goldstein, J. Chem. Phys. 53, 2372 (1970).

${ }^{3}$ N. G. McCrum, B. E. Read, G. Williams, Anelastic and Dielectric Effects in Polymer Solids (Dover, New York, 1991 and [original issue (Wiley, London, 1967)].

${ }^{4}$ H. W. Starkweather, Macromolecules 14, 1277 (1981).

${ }^{5}$ H. W. Starkweather, Macromolecules 21, 1798 (1988).

${ }^{6}$ J. Steinfeld, J. Francisco, and W. Hase, Chemical Kinetics and Dynamics (Prentice-Hall, Englewood cliffs, NJ, 1989).

${ }^{7}$ Side Chain Liquid Crystalline Polymers, edited by C. B. McArdle (Blackie, Glasgow, 1989).

${ }^{8}$ A. N. Donald and A. H. Windle, Liquid-Crystalline Polymers (Cambridge University Press, Cambridge, 1992).

${ }^{9}$ G. Williams, Polymer 35, 1915 (1994).

${ }^{10}$ J. F. Mano, N. M. Alves, J. M. Meseguer Dueñas, J. L. Gómez Ribelles, Polymer 40, 2675 (1999).
${ }^{11}$ R. Zentel, G. Strobl, and H. Ringsdorf, Macromolecules 18, 960 (1985).

${ }^{12}$ F. J. Bormuth and W. Haase, Mol. Cryst. Liq. Cryst. 148, 1 (1987).

${ }^{13}$ S. U. Valerien, F. Kremer, and C. Boeffel, Liq. Cryst. 4, 79 (1989).

${ }^{14}$ G. McMullin, W. G. Scaife, and R. Zentel, 18, 529 (1995).

${ }^{15}$ A. Schönhals, U. Gessner, and J. Rübner, Macromol. Chem. Phys. 196, 1671 (1995).

${ }^{16}$ F. R. Colomer, J. M. Dueñas, J. L. Gómez Ribelles, J. M. Barrales-Rienda, and J. M. Ojeda, Macromolecules 26, 155 (1993).

${ }^{17}$ J. F. Mano and J. J. Moura Ramos, Thermochim. Acta 323, 65 (1998).

${ }^{18}$ J. F. Mano, N. T. Correia, J. J. Moura Ramos, S. R. Andrews, and G. Williams, Liq. Cryst. 20, 201 (1996).

${ }^{19}$ J. F. Mano, Thermochim. Acta 332, 161 (1999).

${ }^{20}$ G. Schaack, Ferroelectrics 104, 147 (1990), and references therein.

${ }^{21}$ M. Manger, S. Lanceros-Méndez, G. Schaack, and A. Klöpperpieper, J. Phys.: Condens. Matter 8, 4617 (1996).

${ }^{22}$ S. Lanceros-Méndez, G. Schaack, M. Köhler, and A. Klöpperpieper, Ferroelectrics 176, 73 (1996).

${ }^{23}$ S. Lanceros-Méndez and G. Schaack, J. Korean Phys. Soc. 32, S850 (1998).

${ }^{24}$ S. Lanceros-Méndez, G. Schaack, and A. Klöpperpieper, Frontiers in Science and Technology, Science and Technology of Ferroelectrics (in press). 